\title{
Stereoscopic PIV aided wake simulation of a catamaran research vessel using a dummy-hull model in a medium size cavitation tunnel
}

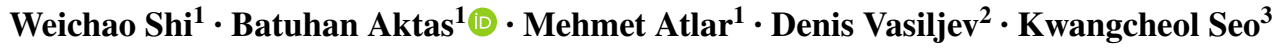

Received: 21 February 2017 / Accepted: 3 October 2017 / Published online: 19 October 2017

(C) The Author(s) 2017. This article is an open access publication

\begin{abstract}
The rising marine environmental concern has recently targeted underwater radiated noise. Amongst various sources present on-board, propeller cavitation noise is known to be the dominant source that may be harmful to marine biodiversity. To be able to minimize anthropogenic noise footprint, full-scale and model-scale test campaigns are the most reliable tools to measure or predict the noise sound pressure level. Within this framework, hydro-acoustic cavitation tunnel experiments carry utmost importance for model-scale tests. Due to limited space of the cavitation tunnel, a shortened dummy-hull is often used, even though the flow passing through the propeller plane of the dummy model does not represent a fully developed wake. This paper presents a wake simulation methodology for a shortened dummy-hull model of Newcastle University research vessel "The Princess Royal" with the aid of stereoscopic particle image velocimetry in Emerson Cavitation Tunnel. With such method, after three iterations sufficient similarity between target wake and simulated wake has been achieved. Adopted approach has been found to be significantly effective in terms of reducing the time and the iterations during wake simulation process.
\end{abstract}

Batuhan Aktas

batuhan.aktas@strath.ac.uk

1 Naval Architecture, Ocean and Marine Engineering, University of Strathclyde, Henry Dyer Building, 100 Montrose Street, Glasgow G4 OLZ, UK

2 IHC Engineering Business Ltd., Stocksfield, Northumberland NE43 7TN, UK

3 Department of Naval Architecture and Ocean Engineering, Mokpo National Maritime University, Haeyangdaehak-ro, Mokpo-si, Jeollanam-do, South Korea
Keywords Wake simulation · Dummy-hull model · Particle image velocimetry (PIV) · Wake screen · Streamline tracing

\section{Introduction}

Underwater radiated noise created by commercial shipping is known to contribute significantly to ambient noise levels of the world seas, especially in the presence of propeller cavitation [1]. Rising environmental concerns for potential harm, which may adversely affect the marine biodiversity, has recently targeted the propeller cavitation noise radiated from the marine merchant fleet [2]. As a result, there is a concentration of attention, co-operative researches and workshop activities at local, European and international levels involving organisational bodies such as IMO, MEPC, ITTC and HTF in the field of underwater radiated noise [3-5]. The rising awareness also resulted in initiation of a number of EU projects. SONIC project is the one that Newcastle University (UNEW) has been involved in. Various full-scale and model-scale experimental campaigns were conducted with UNEW research vessel, the Princess Royal [6]. She was chosen as a benchmark vessel, of which various experimental campaigns have been conducted including: full-scale sea trials; model-scale towing tank tests; dummyhull model cavitation tunnel tests [7]. Recently, within this framework, the dummy-hull model tests were conducted in Emerson Cavitation Tunnel (ECT), UNEW, for the cavitation observation and radiated noise measurement [7]. To accurately reproduce these phenomena in the laboratory, a reliable nominal wake measurement is critical for the tests.

The propeller operates behind the hull and hence its inflow is affected by the hull wake caused by the potential and viscous components of the flow over the hull. The 
propeller is located at the stern of the ship to recoup some of the energy in the hull boundary layer, as well as for improved manoeuvrability properties and protection against damage [8]. Due to the presence of the hull in front and its proximity to the rudder which is behind, the flow in which the propeller operates (the wake) is not uniform. This non-uniformity result in local variations in the angle of attack of the propeller inflow and chord wise extent of cavitation experienced by the propeller and consequently results in higher underwater radiated noise levels $[9,10]$. Thus, wake simulation carries utmost importance within the context of hydro acoustic cavitation tunnel experiments.

The non-uniformity of the wake is divided into components, such as axial, tangential and radial, for the detailed scrutinization of the inflow quality [11]. First of all, the axial wake can affect cavitation dynamics and should be carefully considered during the after-body design of a new ship. The severity of the wake affects the cavitation volume developed and the violence of its collapse both on and off the blade surface. Konno et al. [12] have investigated the importance of the wake using four different severities of wake by altering the spatial gradient of the inflow velocity in the wake shadow area (i.e. where the inflow to the propeller is significantly slowed), whilst the least severe one has a rather gradual change. The corresponding measurements have shown significant acoustic pressure elevations for the wakes with higher velocity gradients, thus demonstrating the importance of the axial wake factor for acoustic tests of the marine propellers.

The tangential component of the wake is generated by the three-dimensional shape of the hull and may be due to the streamline directions of the hull flow or due to an embedded bilge vortex flow. These components change from "with" the blade rotation to "against" the rotation across the 12 o'clock position (top dead centre) and hence also alter the angle of attack to the propeller blade. In combination with the axial wake gradient they can cause the cavitation volume to change more rapidly due to the added variation in the section angle of attack.

To accurately reproduce the behind hull condition, which the propeller is working in, the use of a full ship model is always preferred. However, this method is always regarded as the most expensive option and highly depends on the facilities' capacity. The so-called "Dummy-hull model" is another practical approach that has been used in many medium-size cavitation tunnels including ECT. Amongst the various options of simulating the flow into a propeller, the "Dummy-hull model" approach has the capability to reproduce tangential and radial flow components, along with the standard axial component $[13,14]$. Hence, the wake simulated is of a higher quality in comparison to, e.g. traditional 2D wake screens [15]. In this particular case a single hull dummy-hull model was built to represent our target vessel.
Use of a dummy-hull model for the representation of actual wake distribution in the propeller plane of a fullscale vessel or its geo-sim model, which is known to be "target wake", would require the so-called "wake simulation" task. In cases, where scale-factor of the model is high, Reynolds number difference between model and full-scale ship is significant. The Reynolds numbers for model tests are typically in the range $10^{6}-10^{7}$. Ships are mainly working at Reynolds numbers of $10^{9}$. This difference significantly affects the boundary layer thickness and hence the velocity profile at the propeller plane. To address this problem there is a number of ways to scale the wake ranging from simple empirical formulas to high fidelity numerical simulations $[14,16]$. The most well-known and well established scaling method is the one proposed by Sasajima and Tanaka [17], which is based on a boundary layer wake scaling method where wake fraction is divided into potential and frictional wake components. In the same vein, Hoekstra [18] proposed that each wake component is related to each other. Following these landmark studies more recently Computational Fluid Dynamics are started to be used for smart dummy models that are created based on full-scale CFD simulation wake predictions to modify the model scale dummy hull. By this way dummy hull is modified to induce the wake distribution that is predicted by the full-scale simulations as demonstrated by van Wijngaarden [19]. Whilst, there exists a number of other wake scaling methods it would not be much influential for this particular case as the scale factor is significantly small. In the present case, however, wake simulation is necessary mainly to recover the reduced boundary layer effects due to the truncated parallel mid-body part of the dummy-hull model of the Princess Royal. This could be achieved by fitting suitably selected mesh patches onto the wake screen, which is mainly an iterative procedure and is always time-consuming. Since there is no clear indication where the mesh patches should be added on the wake screen. Therefore, finding the corresponding position from the wake screen to the propeller plane is critical in wake simulation process.

On the other hand, during this process, flow velocimetry technology also plays an important role in these tests. From the most widely used Pitot tube to the advanced particle image velocimetry (PIV), various apparatuses were developed and employed to perform this task. By considering the intrusive nature of some kinds of devices, technologies like PIV and LDA (Laser Doppler Anemometry) does not interfere with the flow field being positioned outside the measuring field. In comparison to LDA, which is a single point measurement, PIV has the capability to measure a plane or a volume with one measurement [20,21].

This paper presents the wake simulation for the dummy model with the aid of stereoscopic PIV (SPIV). A truncated dummy-hull model used for the wake simulation is not 
enough to completely developed flow boundary layer as for a general ship model. Therefore, construction of a wake screen is required to reproduce similar conditions for the propeller to perform its cavitation and noise tests. Before any modification to the dummy-hull model's wake, the streamline from the wake screen was traced by soft threads. This approach allows to see the cross-flow to figure out the corresponding position from the wake screen to the propeller plane. Hence, the construction of wake screen is more effectively controlled. As a result, a sufficient similarity between target wake and the simulated wake was achieved within three wake mesh iterations.

Within this framework, following this introduction, Sect. 2 of the paper describes the experimental facilities and dummy hull model methodology. In Sect. 3, wake simulations methodology is presented including PIV acquisition and handling (Sect. 3.1), wake screen construction (Sect. 3.2) and streamline tracing methodology (Sect. 3.3). Section 4 presents the results and discussions regarding the conducted measurements and efficiency of the adopted methodology. Finally, Sect. 5 presents the overall conclusions obtained from the investigation.

\section{Description of the facility and the testing model}

\subsection{Description of ECT}

The experiments were conducted in the Emerson Cavitation Tunnel (ECT) at the Newcastle University. The tunnel is a medium size propeller cavitation tunnel with a measuring section of $1219 \times 806 \mathrm{~mm}$ (width $\times$ height). The speed of the tunnel water varies between 0.5 and $8 \mathrm{~m} / \mathrm{s}$. The general topology and technical information about the facility is shown in Fig. 1 and Table 1. More detailed information about the tunnel and the test facilities are given by Atlar [22].

\subsection{Description of the used dummy-hull model}

The scale factor of 1:3.5 was set by taking various limiting factors into account, e.g. associated with the undesirable blockage effect, reasonable Reynolds number range for the consequent propeller testing, minimising the scale effects whilst meeting size limitation. Due to a slim hull body, the model at this scale appeared to be too long $(5.39 \mathrm{~m})$ to fit the tunnel's test section and had to be truncated down to $3 \mathrm{~m}$ to be fitted into the testing section. The truncation is preferably carried out in the parallel mid-section between the properly represented fore and aft sections, as shown in Fig. 2. However, such approach forced to take an assumption of a

Table 1 Technical information of ECT

\begin{tabular}{ll}
\hline Description of facility & Vertical plane, closed circulation \\
\hline Test section size $(L \times B \times H)(\mathrm{m})$ & $3.10 \times 1.22 \times 0.81$ \\
Test section area $\left(\mathrm{m}^{2}\right)$ & 1.008 \\
Contraction ratio & 4.271 \\
Main pump power $(\mathrm{kW})$ & 300 \\
Main pump rotation speed $(\mathrm{RPM})$ & 294 \\
Impeller diameter $(\mathrm{m})$ & 1.4 \\
Maximum velocity $(\mathrm{m} / \mathrm{s})$ & 8 \\
Absolute pressure range $\left(\mathrm{kN} / \mathrm{m}^{2}\right)$ & $7.6(\mathrm{~min})-106(\max )$ \\
Cavitation number range & $0.5(\mathrm{~min})-23(\max )$ \\
Model propeller size $(\mathrm{mm})$ & $150-400$ \\
\hline
\end{tabular}

Fig. 1 General topology of ECT

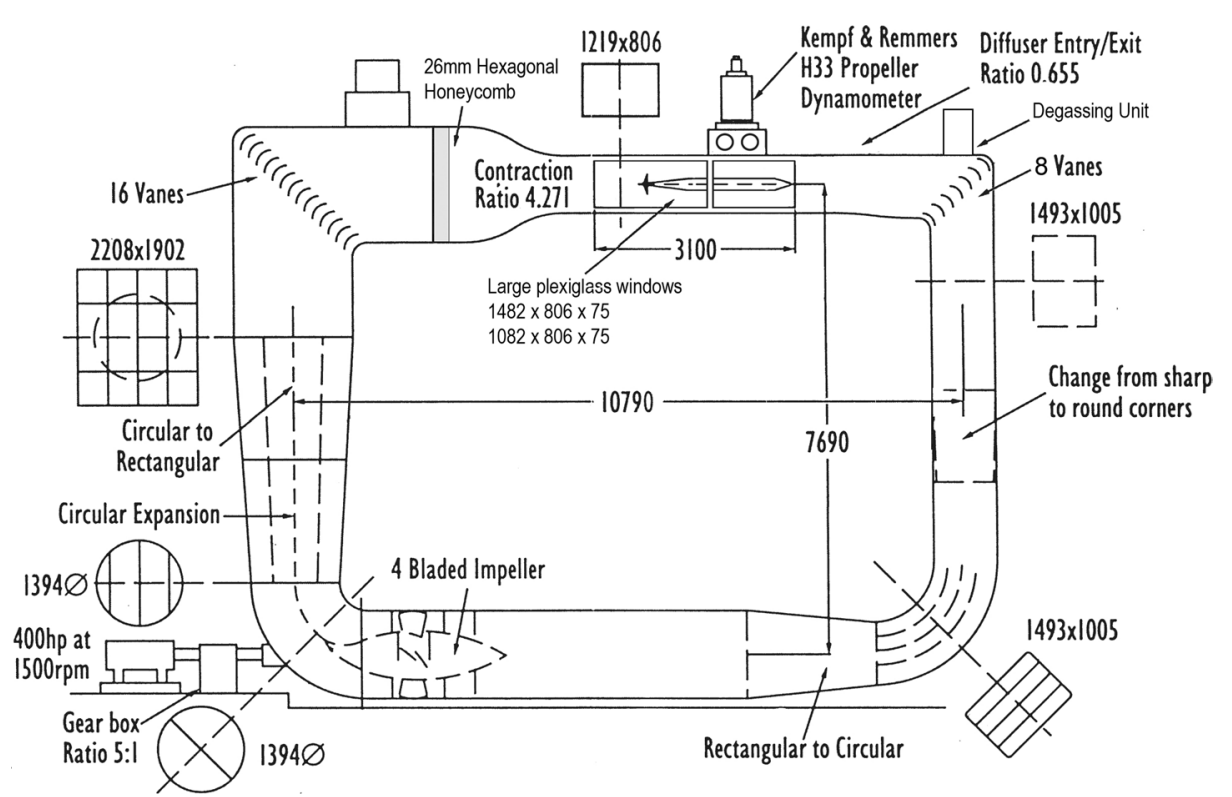




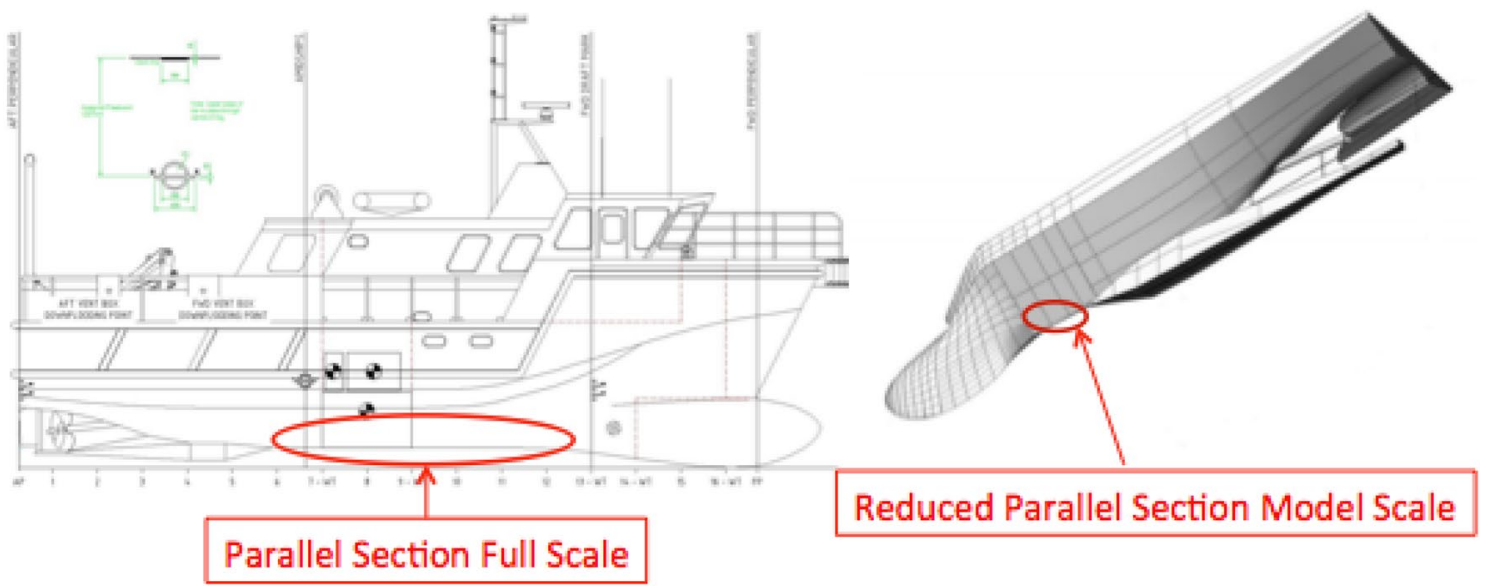

Fig. 2 Dummy-hull philosophy

Table 2 Full scale vessel and hull model general particulars

\begin{tabular}{llll}
\hline & $\begin{array}{l}\text { Full scale } \\
\text { (beginning of } \\
\text { trials) }\end{array}$ & $\begin{array}{l}\text { Full scale } \\
\text { (end of tri- } \\
\text { als) }\end{array}$ & $\begin{array}{l}\text { Model scale UNEW (dummy- } \\
\text { hull model of starboard hull) }\end{array}$ \\
\hline Length overall & 18.88 & 18.88 & 3.007 \\
Draft at forward perpendicular, TF (m) & 1.745 & 1.72 & 0.566 \\
Draft at aft perpendicular, TA (m) & 1.845 & 1.82 & 0.495 \\
Propeller distance from aft perpendicular & 0.9 & 0.9 & 0.262 \\
\hline
\end{tabular}

fully symmetric flow between the demi-hulls. Hence, only the starboard demi-hull was considered in representing fully symmetric twin-hull configuration of the Princess Royal.

The general specification of the hull model used during the experiments and its comparison with the hydrostatic and general particulars of the full-scale vessel are given in Table 2. The data includes two sets of ship particulars. This is necessary due to the fact that the full-scale sea trials of the Princess Royal in September 2013 were conducted over 3 consecutive days meaning the fuel consumptions and various other changes on-board resulted in slight changes in the running conditions of the vessel, especially for trim and sink. This also plays an important role in wake simulation.

The wake distribution data of the starboard demi-hull achieved from the wake surveying task conducted on a 1:5 scale full-model of the Princess Royal in the Ata Nutku towing tank of the Istanbul Technical University (ITU) was taken as the target wake for the wake simulation task. The wake data was obtained by utilising a computer-controlled 5-holed pitot rake fitted to the aft end of the model. Figure 3 shows the target wake distribution [23]. The nominal axial wake velocity ratio is derived by the non-dimensional axial velocity ratio. This calculated by dividing the local axial velocity $\left(V_{\mathrm{x}}\right)$ by the ship's advance speed $\left(V_{\mathrm{s}}\right)$.

During the wake tests, the flow speed was set to the corresponding ship speed of $V_{\mathrm{s}}=15.2$ knots using the Froude

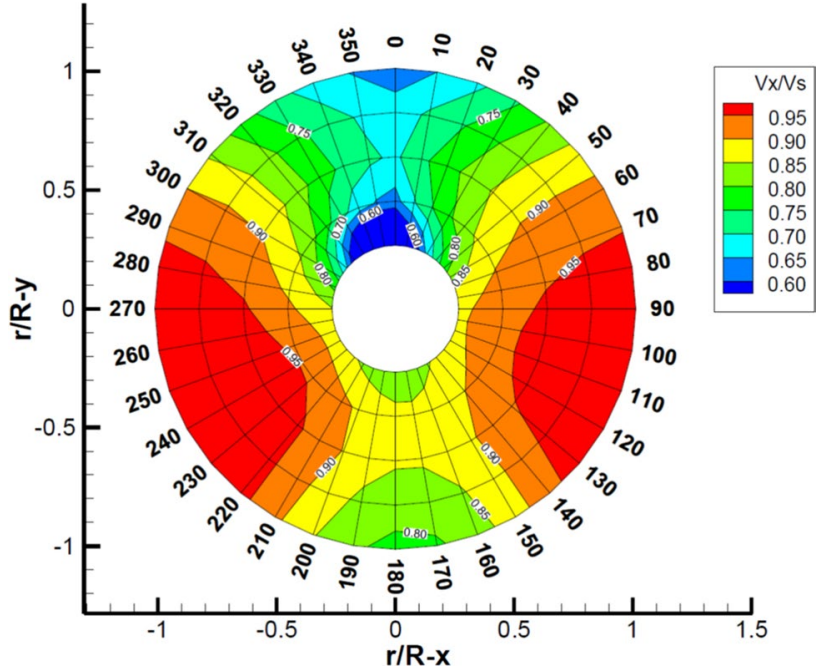

Fig. 3 Nominal wake test in ITU where $V_{\mathrm{x}}$ is the local axial velocity and $V_{\mathrm{s}}$ is the ship's advance speed

number similarity which corresponds to approximately $3.5 \mathrm{~m} / \mathrm{s}$. The target wake data are presented for different nondimensional radii of the propeller $(r / R)$ along " $x$ " $(r / R-x)$ and $y$ axis. Therefore, the target wake measurements are carried out at conditions corresponding to Reynolds Number of $9.28 \times 10^{6}$ calculated using Eq. 1 : 
Table 3 Details about the stereoscopic PIV

\begin{tabular}{ll}
\hline Laser & New wave pegasus \\
\hline Light sheet optics & $80 \times 70$ high power Nd:YAG light sheet series \\
Synchronizer & NI PCI-6601 timer board \\
Camera & NanoSense MK III \\
Sensor size & $1280 \times 1024$ pixels \\
Maximum capture frequency & $1000 \mathrm{~Hz}$ \\
Per-cavity energy at $527 \mathrm{~nm}$ at $1 \mathrm{kHz}$ & $10 \mathrm{~mJ}$ \\
Maximum images & 3300 \\
Calibration target & Multi-level $270 \times 190 \mathrm{~mm}, 2$ nd level-4 \\
Seeding particles & Talisman 30 white 110 plastic powder
\end{tabular}

$R e=\frac{V \times L}{v}$

where $V$ is the inflow velocity, $L$ is the length of the model and $\nu$ is the kinematic viscosity of tunnel water.

\section{Wake simulation method}

\subsection{PIV data acquisition and handling}

Flow measurements for wake simulations are traditionally carried out with pitot tubes. However, due to the intrusive nature of this technique, more recently such measurements are conducted with laser based techniques such as Laser Doppler Anemometry (LDA) or particle image velocimetry (PIV). For the present study, flow measurements were done using SPIV. The SPIV device used in the ECT is a Dantec Dynamics Ltd system and a summary of its technical details are given in Table 3.

A typical SPIV system includes: a laser illumination system; two high-speed PIV cameras; and a timer board to synchronize the whole measuring system. A Pegasus-PIV laser system, which consists of two IR laser heads, can generate high-energy laser light to illuminate the measuring area for double-frame image capturing task. And a closed laser light guide arm transfers the light beam to an $80 \times 70$ light sheet optic to produce a light sheet to illuminate a planar section for the measurement. In this case, the velocity of seeding particles passing through the light sheet is measured. The thickness of the light sheet is tuned to be $4 \mathrm{~mm}$ thick to ensure the highly seeded and well-illuminated interrogation area. Two high-speed cameras are used to capture the image from both sides of the light sheet and a traverse system is also included to move the light sheet and the cameras. The setup of the SPIV system in the ECT is shown in Fig. 4.

To calibrate the SPIV system, a multi-level $270 \times 190 \mathrm{~mm}$ calibration target had to be used, and therefore, installed beside the propeller shaft at the wake

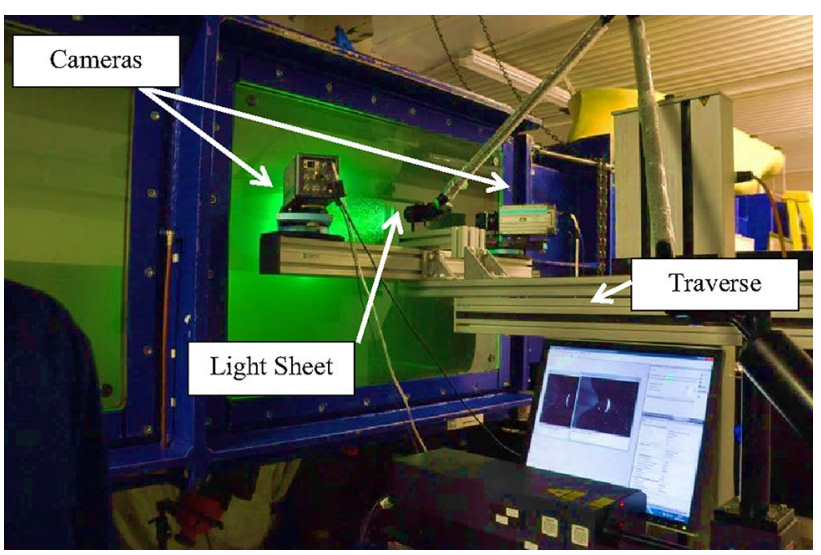

Fig. 4 Setup of stereoscopic PIV

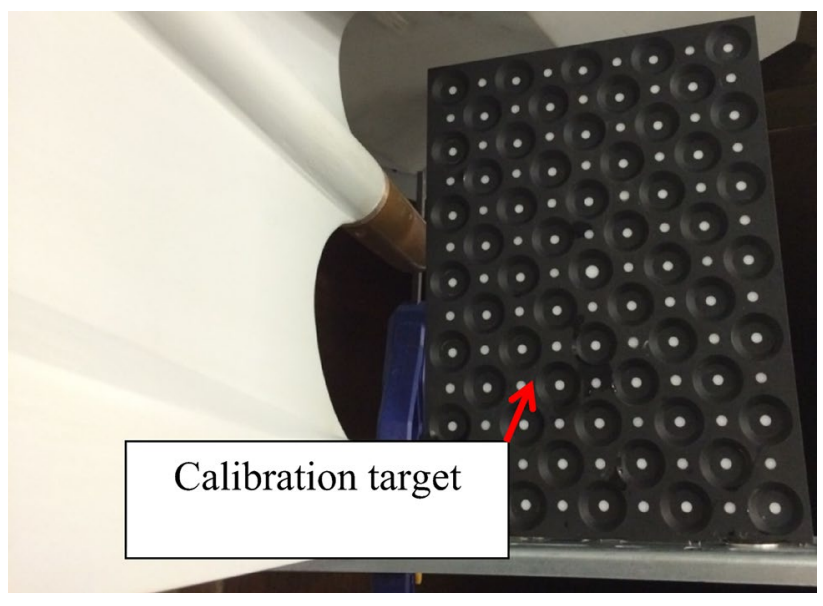

Fig. 5 Calibration target installed on the dummy-hull model

plane, as shown in Fig. 5. In the calibration process, it is generally assumed that the $X Y$-plane where $Z=0$ corresponds to the centre of the light sheet, but in practice this assumption may not hold since it can be very difficult to properly align the calibration target with the light sheet. To improve the accuracy of the calibration, the initial calibration results were needed to be refined by combining the 
images acquired simultaneously from both cameras with a built-in calibration refinement function. The comparison is presented in Fig. 6 with the coordinate system definition. The green grid the initial calibration results, whereas the red grid represents the refined calibration results.

Throughout the measurements 100 double frame image pairs were captured with a $100 \mathrm{~Hz}$ frequency and $240 \mu$ s delay between two frames. A sample of the captured images and a sample of the normalized cross-correlation map between two frames are presented, respectively, in Figs. 7 and 8. As shown in Fig. 8, the peak value can be well detected which assured the image quality and the seeding density are guaranteed for the current measurement.

With the sampled images, the adaptive PIV analysis was applied to acquire the 2D velocity vector, following be the range validation and moving average validation to eliminate the invalid data. Afterwards, by combining the calibration results and the 2D image data, the SPIV data could be achieved. Finally the results of the 100 samples were averaged to obtain the final SPIV result, as shown in Fig. 9 which show the axial velocity distribution tested at $2 \mathrm{~m} / \mathrm{s}$ and the standard deviation of the axial velocity. Due to the block of the light sheet by the propeller shaft and the image aberration caused by the large observation angle, data quality at the left side (starboard side) is relatively poor. Therefore, given that the dummy model is a symmetrical model to the mid plane, the right side (port side) of the propeller shaft was regarded as the valid area which is marked in the blue frame as shown in Fig. 9. The data was then extracted and mirrored to form the whole wake and then normalised against the surrounding velocity as shown in Fig. 10.
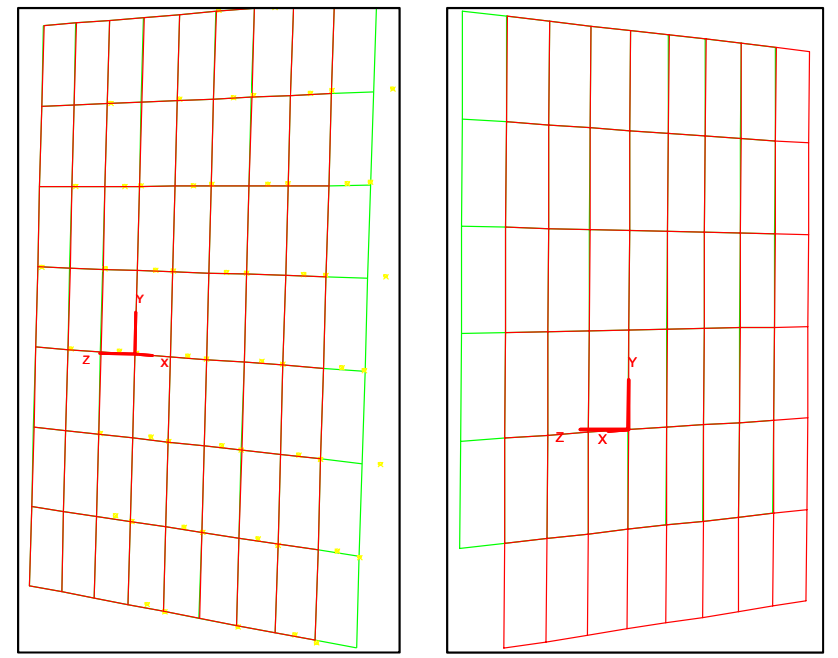

Fig. 6 Calibration result (left image for the forward camera and right image for backward camera)

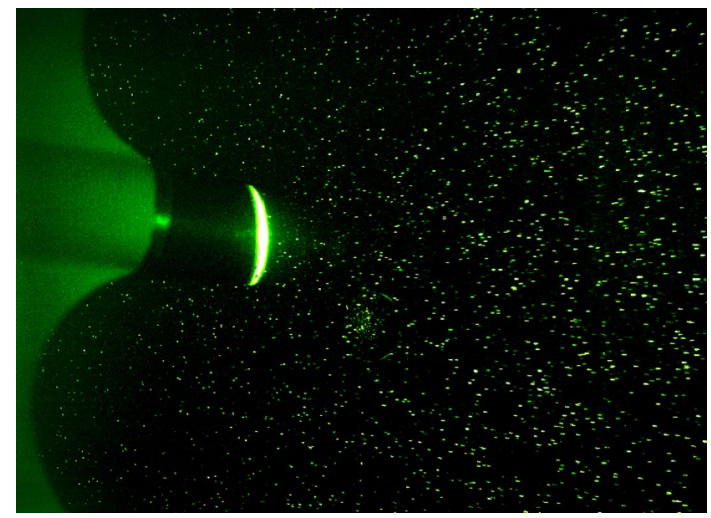

Fig. 7 An example of PIV images

\subsection{Wake screen construction}

In the present case, based on the accumulated knowledge in the ECT, a "base wake screen", which was made of $10 \times 10 \mathrm{~mm}$ size of wire mesh grids, was used. The base screen was laid over a steel framework, which was located in a transverse plane at a distance of 1.5 propeller diameter upstream of the propeller's hub centre, as shown in Fig. 11.

To achieve a reasonable similarity between the target wake and simulated wake iterations were conducted and wake screens patches of different sizes were overlaid on the base mesh. The details of the patches following the final iteration of the wake simulation for starboard side are shown in Fig. 12, while the presentations of the wake simulation results following each iteration and in comparison are given in the following section.

\subsection{Streamline tracing methodology}

To make the wake screen construction more efficient, a streamline tracing experiment was conducted to figure out the corresponding position from the wake screen to the wake

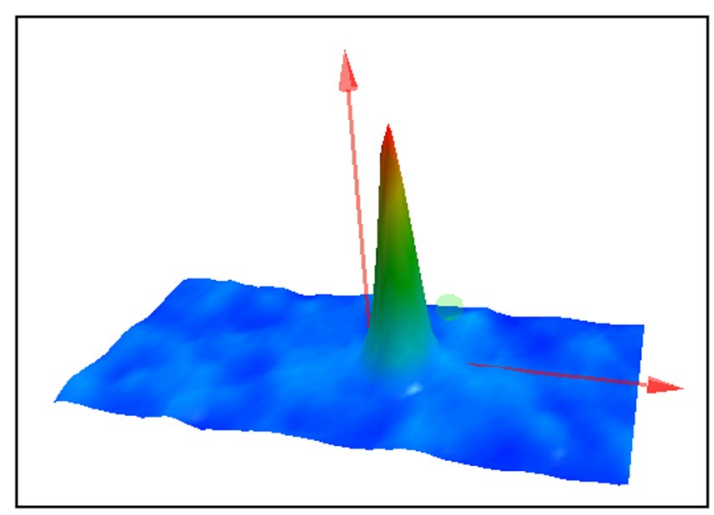

Fig. 8 Normalised cross-correlation map 
Fig. 9 Example of stereoscopic PIV result with contour plot at $2 \mathrm{~m} / \mathrm{s}$ (top: axial velocity $V_{\mathrm{x}}$, bottom: standard deviation of $V_{\mathrm{x}}$ )
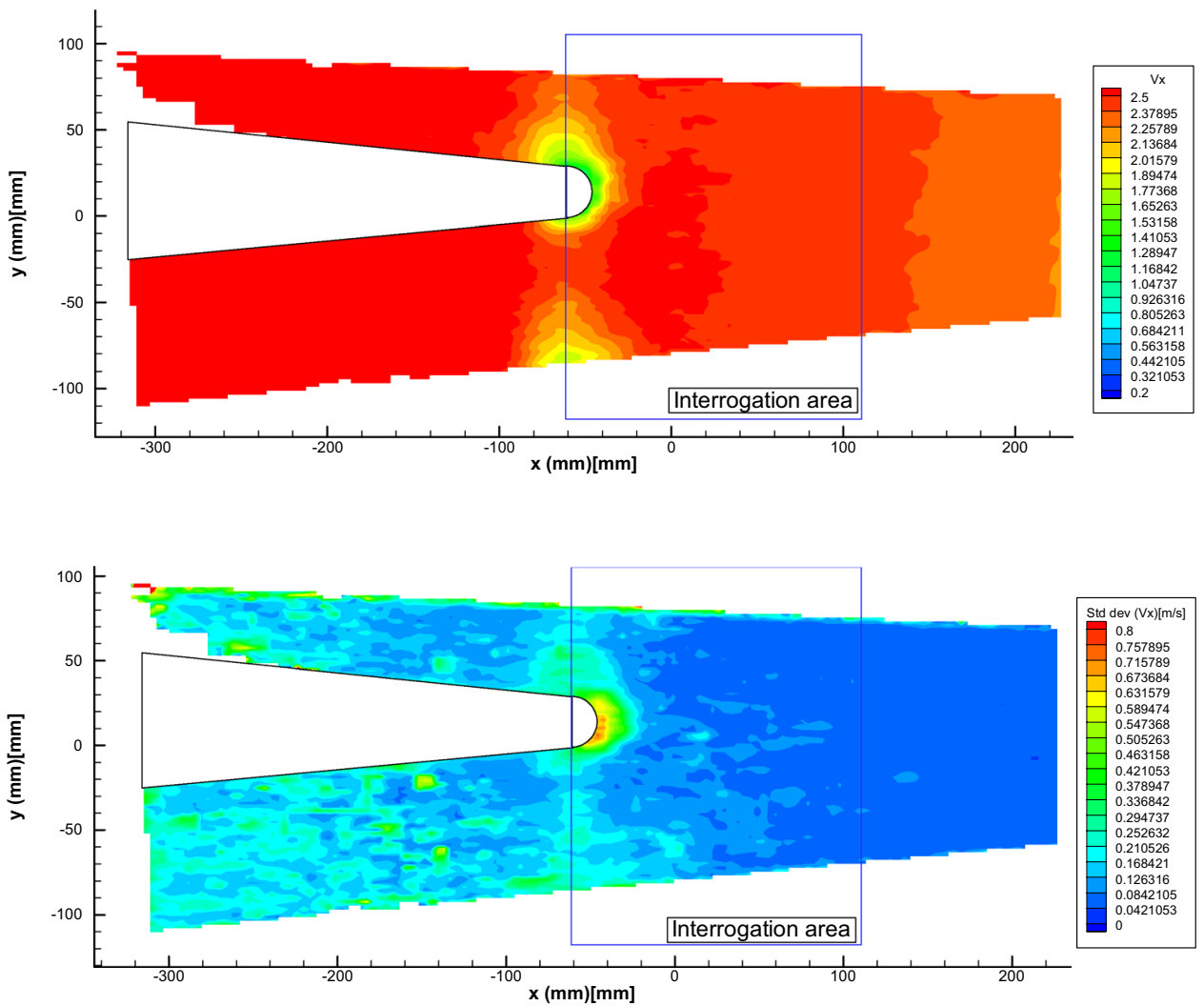

Nominal wake of the bare dummy model $(2 \mathrm{~m} / \mathrm{s})$

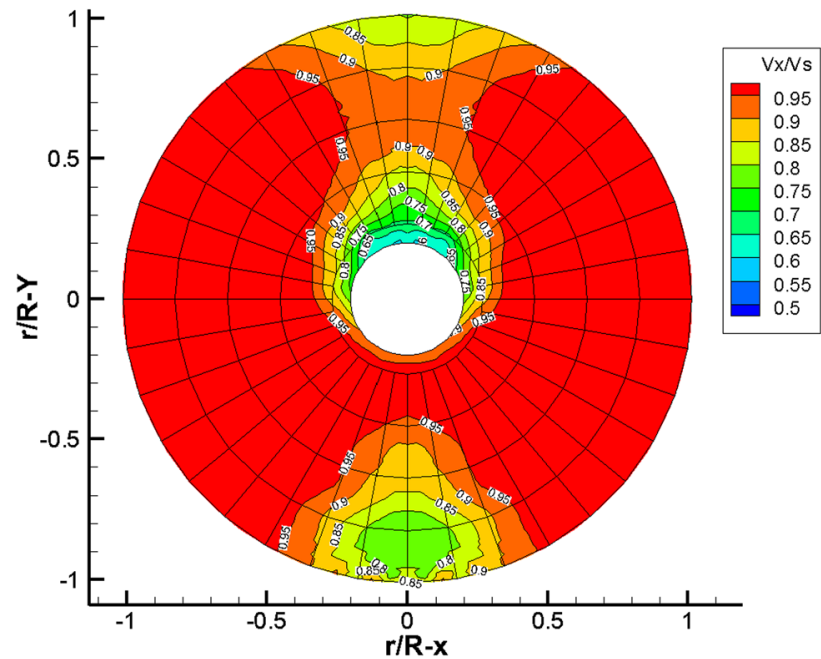

Fig. 10 Nominal wake of the bare dummy model at $2 \mathrm{~m} / \mathrm{s}$ where $V_{\mathrm{x}}$ is the local axial velocity and $V_{\mathrm{s}}$ is the ship's advance speed

plane. To trace the streamlines visually sufficient numbers of soft threads with different colours were attached to the base mesh at strategic positions, shown in Fig. 13. The projections of the trailing ends of these threads is a very useful practical guide for strategically positioning of wire mesh grids on the base mesh, as shown in Figs. 14 and 15.

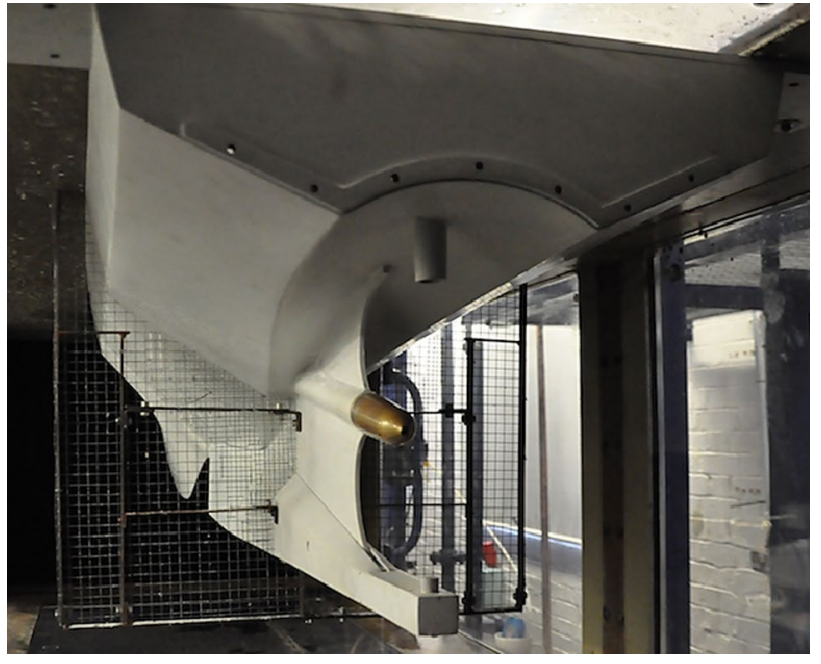

Fig. 11 Wake screen construction

Although the use of the tracing threads was very helpful, the projection of their ends in the wake plane required accurate photographic techniques. At this point the use of the SPIV provided another advantage to reconstruct the images of the threads in 3D and hence accurately locate their ends in the wake plane. This was practical due to a strong scattering of the laser sheet from the soft threads whose projection in the wake plane can be easily identified by the cameras, as 

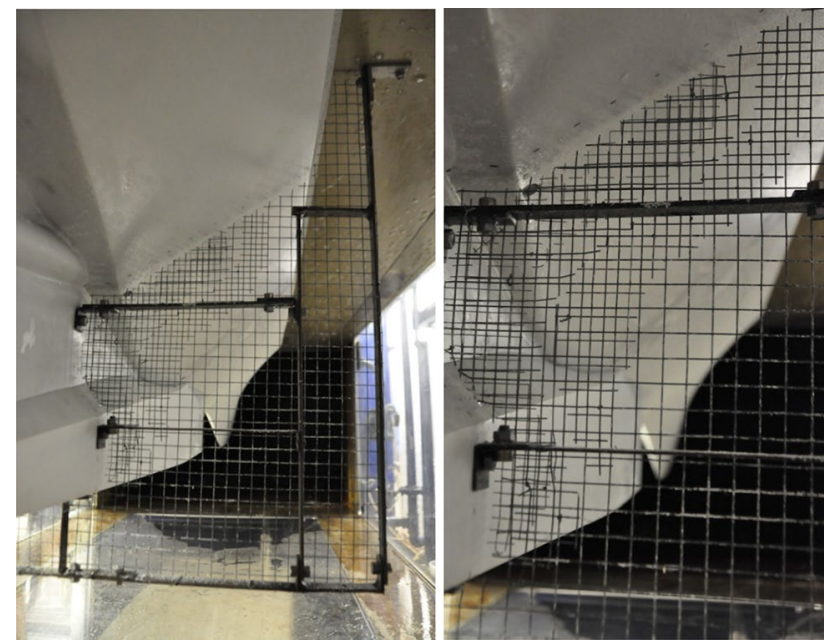

Fig. 12 Wake simulation final attempt (details of the applied patches)

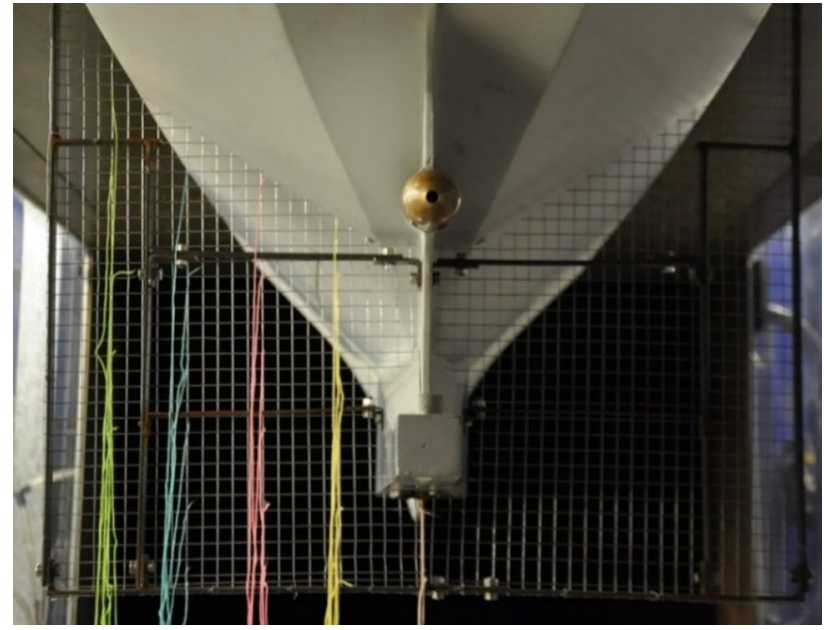

Fig. 13 Streamline tracing threads

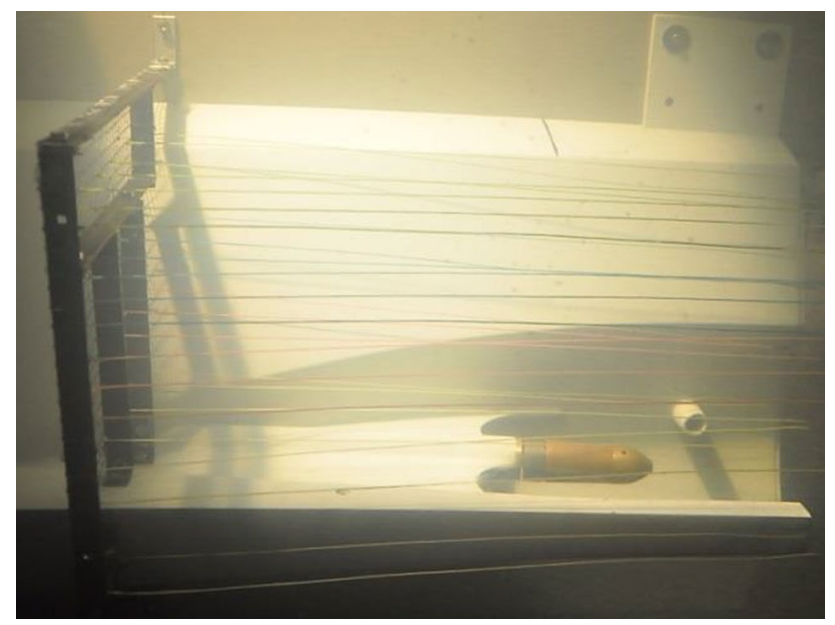

Fig. 14 Bottom view of the tracing threads

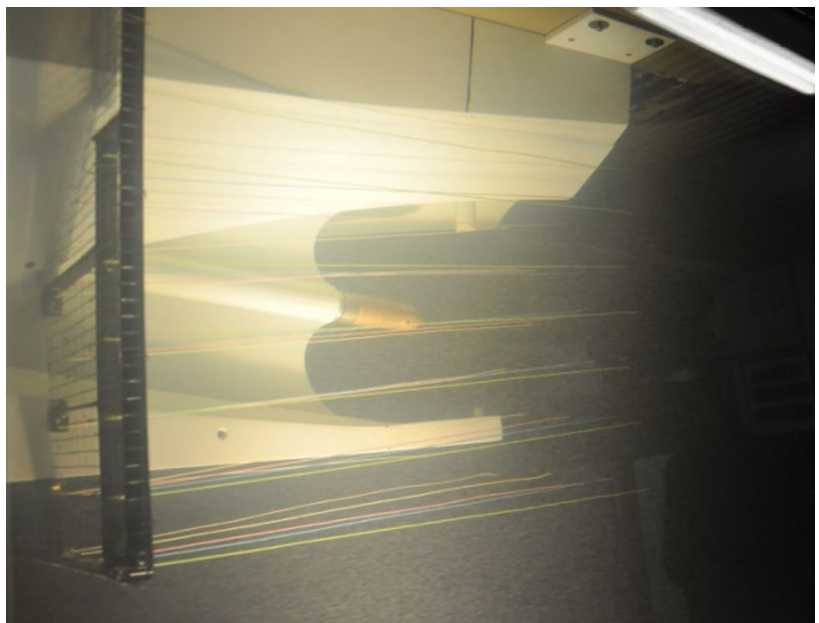

Fig. 15 Side view of the tracing threads

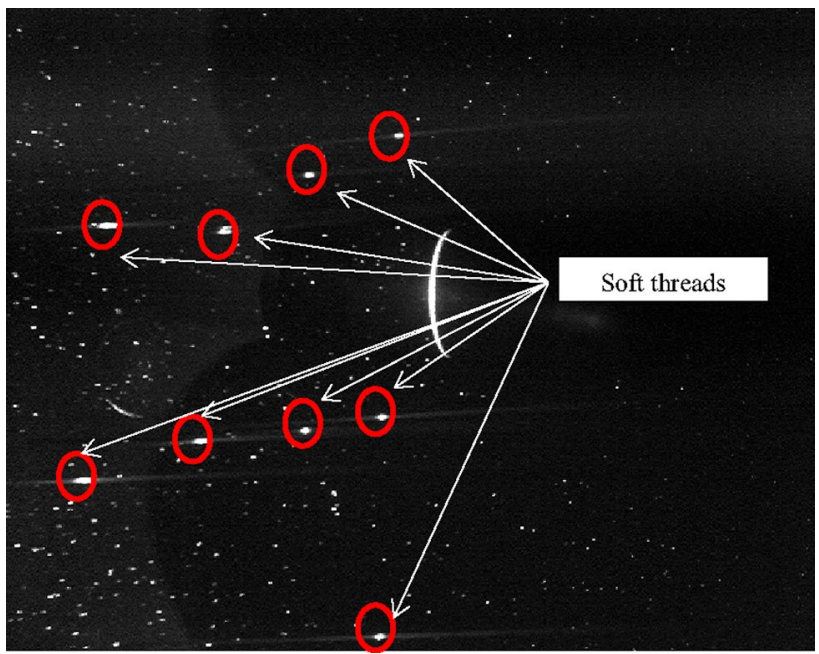

Fig. 16 Image for streamline tracing

shown in Fig. 16. Furthermore, since the velocities near the soft threads were much slower than the other areas, after the processing of the images, the projections of the ends of the soft threads can be clearly identified in the wake field plot as shown in Fig. 17. As shown in this figures the ends of the six soft threads projected in the propeller plane with two threads at around $r=0.64 R$ radius and four threads at around $r=1.013 R$ area.

While the first intention was to use threads as a reference to determine the nature of the flow towards the propeller plane. It was observed from Figs. 12 and 13 that precise location cannot be visually found by eyes. However, the use of the PIV could capture the presence of these soft threads, these images were thence reconstructed 3 dimensionally to determine the precise locations and hence mesh patches on wake screen can be added effectively. Using Fig. 17 in 


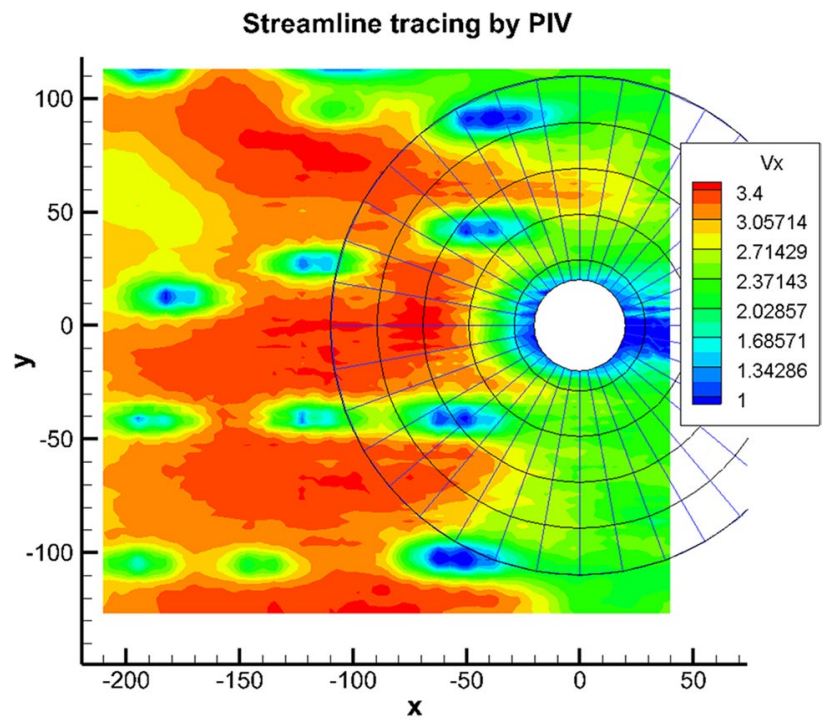

Fig. 17 PIV for streamline tracing

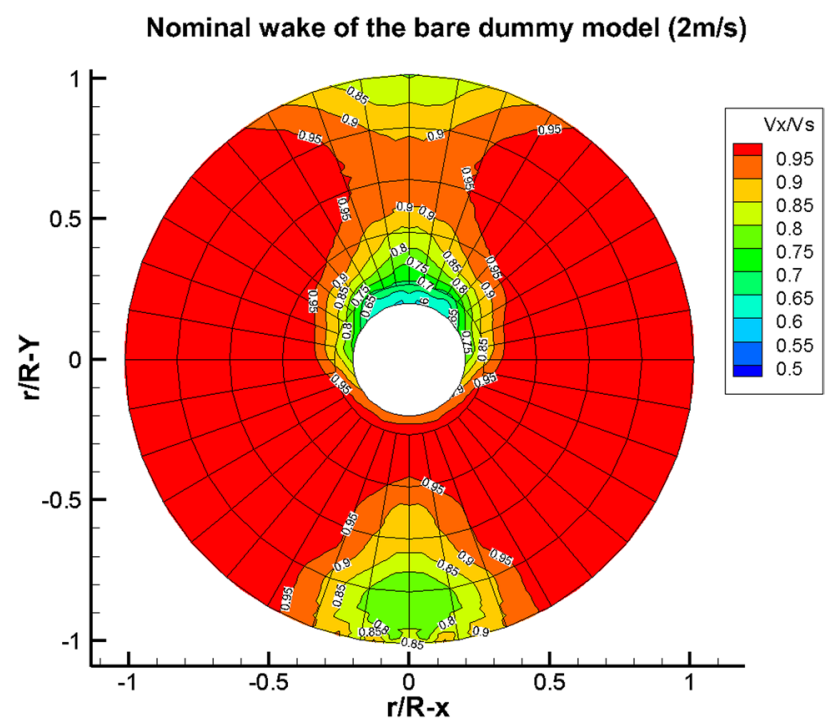

Fig. 18 Nominal wake of the bare dummy-hull model, $2 \mathrm{~m} / \mathrm{s}$ where $V_{\mathrm{x}}$ is the local axial velocity and $V_{\mathrm{s}}$ is the ship's advance speed

particular, it was significantly easier to determine where to add or remove mesh patches on the wake screen.

\section{Result and discussion}

\subsection{Wake distribution of bare dummy-hull under different Reynolds numbers}

Before fitting any wake screen, the initial wake velocity measurements were performed with the bare dummy-hull. These initial measurements were conducted at 2,3 and $4 \mathrm{~m} / \mathrm{s}$

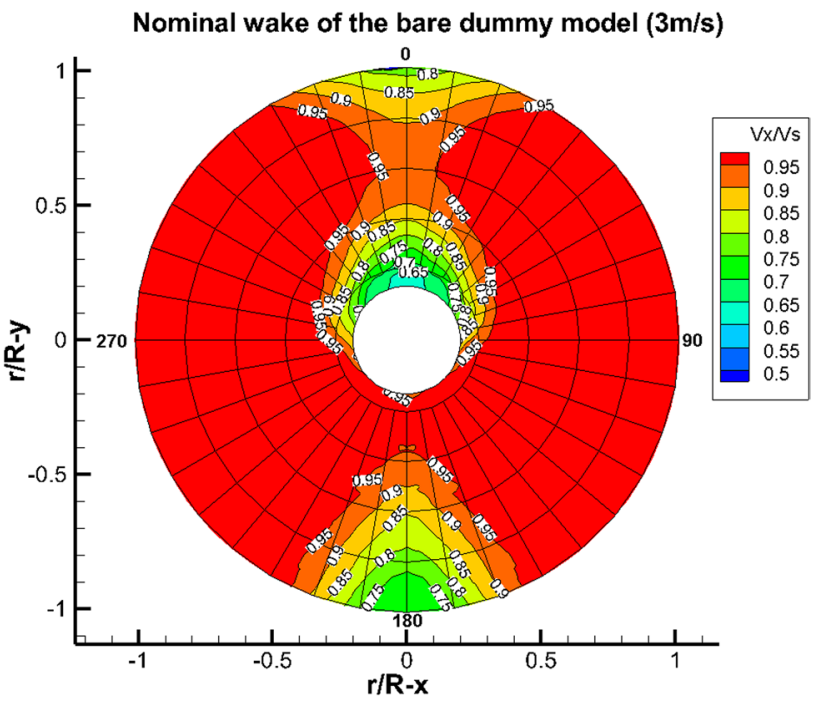

Fig. 19 Nominal wake of the bare dummy-hull model, $3 \mathrm{~m} / \mathrm{s}$ where $V_{\mathrm{x}}$ is the local axial velocity and $V_{\mathrm{s}}$ is the ship's advance speed

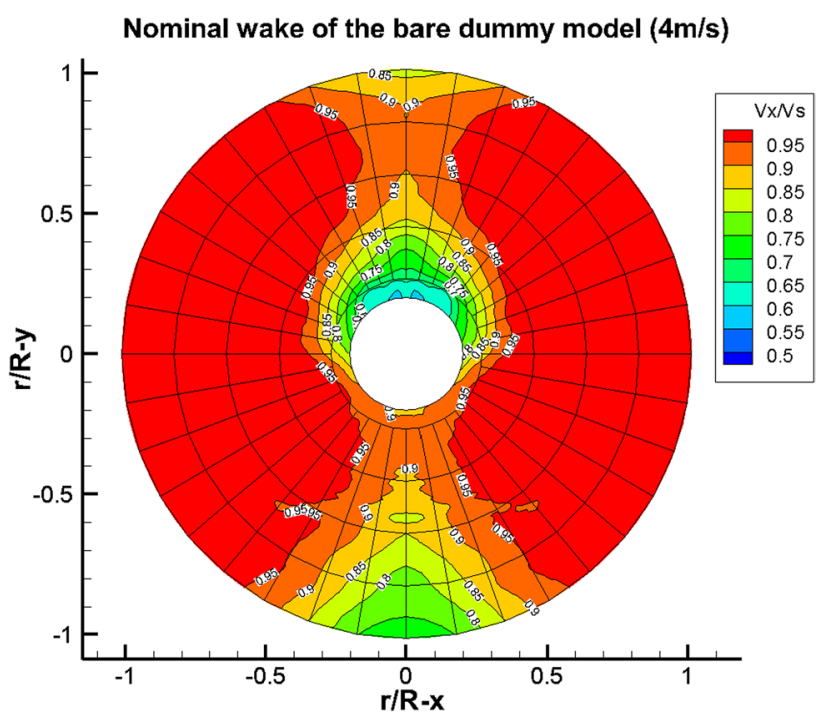

Fig. 20 Nominal wake of the bare dummy-hull model, $4 \mathrm{~m} / \mathrm{s}$ where $V_{\mathrm{x}}$ is the local axial velocity and $V_{\mathrm{s}}$ is the ship's advance speed

tunnel inflow velocities to check the Reynolds number influence and also to see the difference with the target wake.

The measured wake results for the bare dummy-hull are presented in Figs. 18, 19 and 20. Reynolds number of the carried out velocity range with the bare hull varied from $4.62 \times 10^{6}$ to $9.23 \times 10^{6}$ for 2 to $4 \mathrm{~m} / \mathrm{s}$ which were calculated using Eq. 1. According to the measurement results, the wake shadow area is growing with increasing velocity and hence the increasing Reynolds number but within a limited magnitude. As expected, the comparison of the wake data for the bare model with the target wake (in Fig. 3) displayed 
considerable disagreement as such mainly requiring slowing down of the dummy-hull wake velocities due to truncation of the parallel mid-body length.

\subsection{Result of wake simulation}

With the guidance obtained from the streamline tracing experiment, which provided physical relationship between wake screen plane and actual wake plane where the axial velocities are compared, the necessary wake screen patches of different sizes were overlaid on the base mesh and iterative simulations were conducted. For the following noise and cavitation test which would be conducted mostly under $3 \mathrm{~m} / \mathrm{s}$, the wake screen modifications were conducted under $3 \mathrm{~m} / \mathrm{s}$. The simulated axial wake velocities were measured in the wake plane using the SPIV and then compared with the target wake. In the simulation exercise more emphasis were put on the accuracy of the wake velocities at higher radius $(r>0.8 R)$ and at the top dead centre (TDC) and the bottom dead centre (BDC) where the most significant wake shadow was observed.

The comparative plots of the normalized axial wake data $\left(V_{\mathrm{x}} / V_{\mathrm{s}}\right)$ for varying angular positions are presented in Figs. $21,22,23,24$ and 25 for different radii, $r / R=0.267$, $0.453,0.64,0827$ and 1.013 , respectively. In these figures, the wake data is compared to the target wake and bare dummy-hull wake as well as to the results of the three consecutive wake simulations using the wire meshes on the dummy-hull. The angular positions in the figures were selected from $180^{\circ}$ (BDC) to $360^{\circ}$ (TDC), this corresponded to the port side of the target wake which was fitted with the pitot rake during the target wake measurements. As one would appreciate that the target wake measurements were taken with the full-model and hence a slight asymmetry in

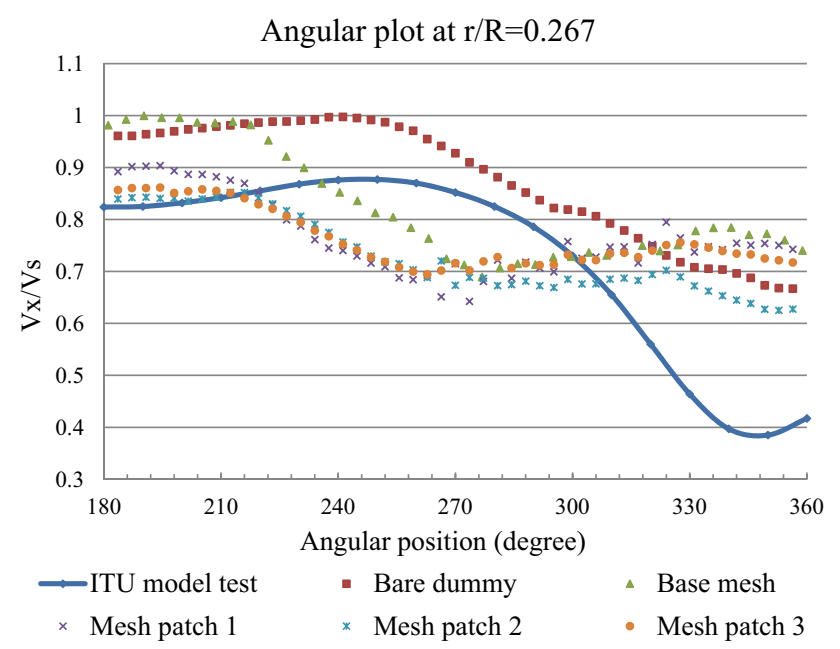

Fig. 21 Comparative angular plot of wake velocities at $r / R=0.267$

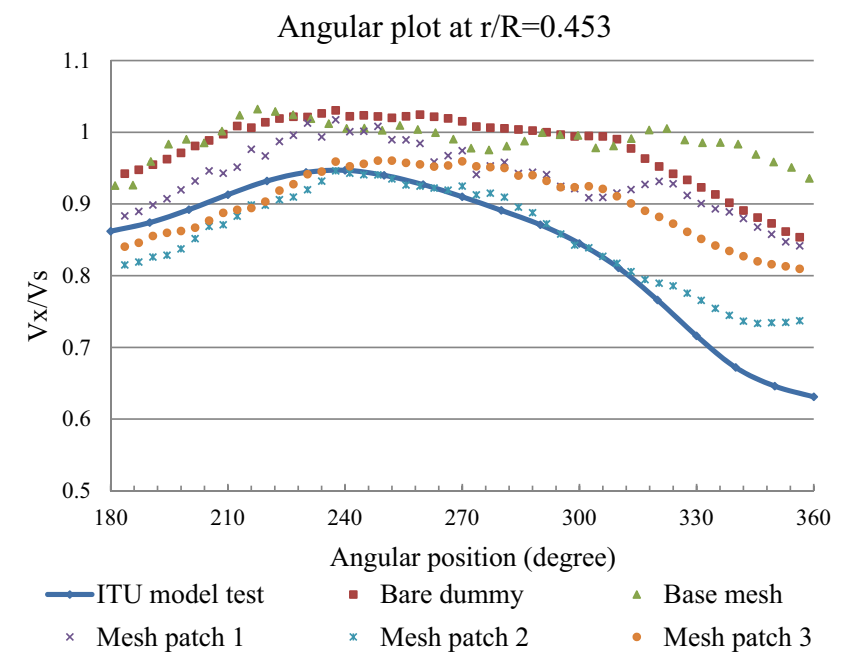

Fig. 22 Comparative angular plot of wake velocities at $r / R=0.453$

the target wake data was observed as shown in Fig. 3. However, this asymmetry was not captured in the wake simulations for the tunnel tests due to the symmetric dummy-hull model.

As shown in Figs. 21, 22, 23, 24 and 25 the improvements in the simulated wake relative to the target wake data are obvious with the consecutive iterations. The agreements for radii $r / R>0.453$ are particularly encouraging to meet the expectations for better cavitation and noise validations.

Figure 26 below, presents the target wake from ITU tests on the left and the simulated wake at ECT on the right. The measurements after the addition of the 3rd mesh patch has shown satisfactory resemblance of the target wake particularly in the outer radii.

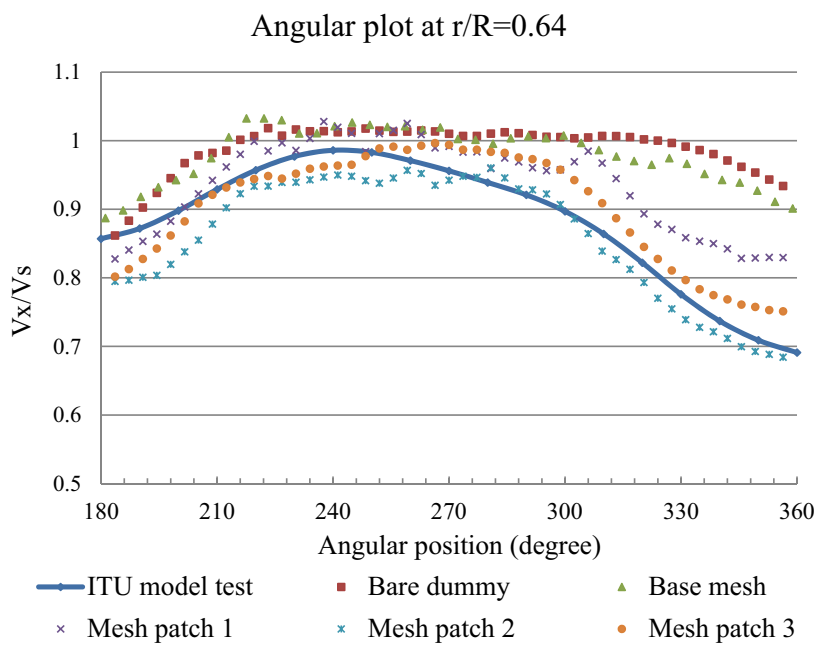

Fig. 23 Comparative angular plot of wake velocities at $r / R=0.64$ 


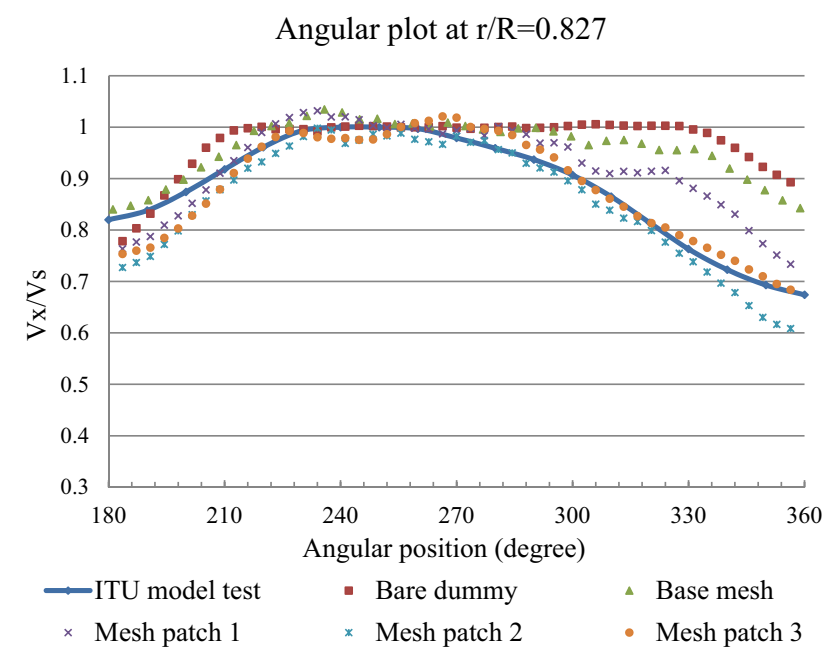

Fig. 24 Comparative angular plot of wake velocities at $r / R=0.827$

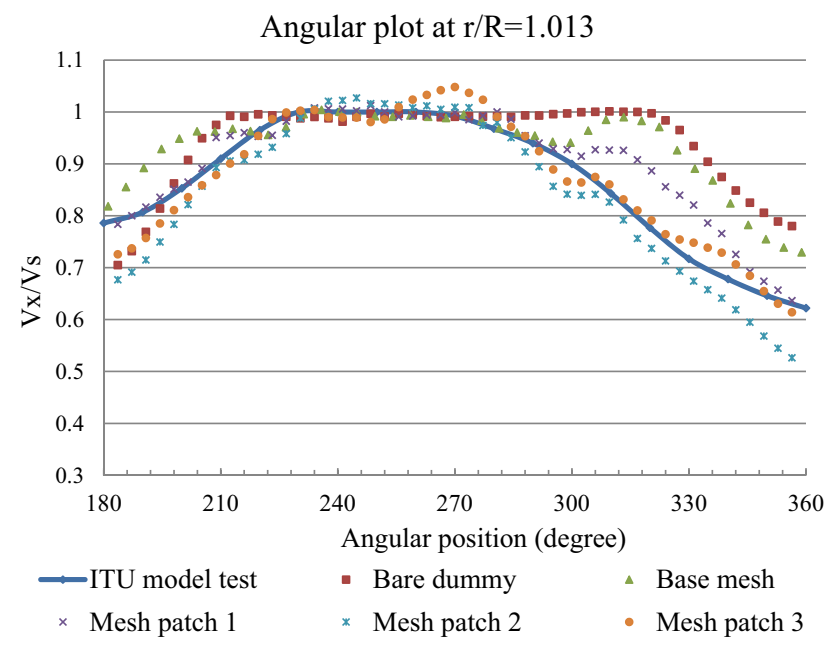

One of the major concerns regarding the wake simulation using a combination of a dummy model and wake screen is their potential impact on the upstream turbulence. The presence of mesh patches is known to stimulate turbulence intensity of the flow $[24,25]$. This may consequently result in premature cavitation inception and elevation of background noise levels of the cavitation tunnel. The acoustical impact of the turbulence intensity is cured by the background noise correction [26].

\subsection{Flow development around the wake plane}

To fully understand the impact caused by the presence of the rudder, another group of measurements have been conducted with the rudder at $3 \mathrm{~m} / \mathrm{s}$. The comparison between the target wake and the simulated wake is presented in the following charts (Figs. 27, 28, 29, 30, 31). Accordingly, the presence of rudder does not pose great impact on the wake distribution. The simulated wake is still closed to the target wake distribution. Therefore, this wake screen and dummy model is qualified for the following propeller cavitation and noise measurement.

Finally, to further investigate the development of the flow along the propeller shaft, further wake field measurements were taken at two vertical planes upstream of the propeller plane at $x / D=-0.23,-0.46$ and one vertical plane downstream of the propeller at $x / D=+0.23$. The results of these measurements are shown in Fig. 32 to give a 3D view of the wake development where the gradual effect of the wake shadow at the TDC and that of the skeg in the BDC can be appreciated. Furthermore, the effect of the rudder in the downstream plane can be clearly seen.

Fig. 25 Comparative angular plot of wake velocities at $r / R=1.013$
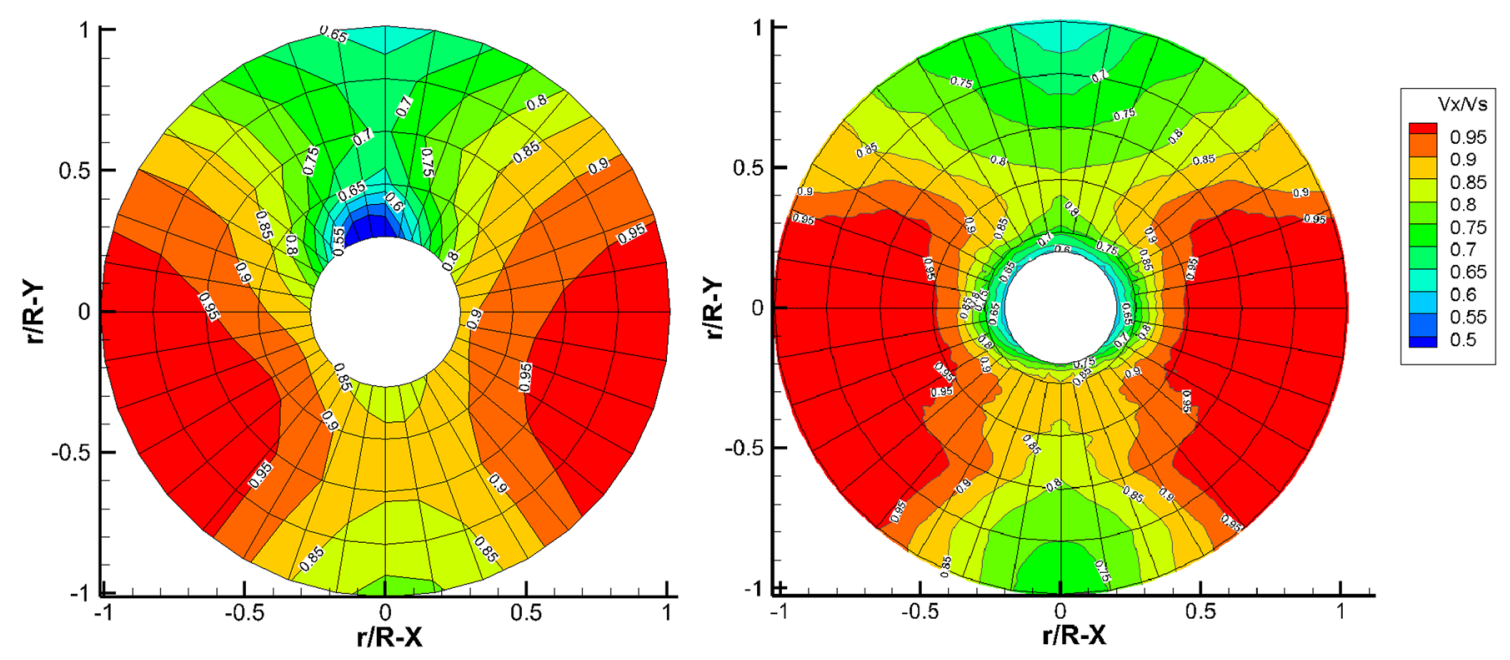

Fig. 26 Contour plots of target wake (left) and simulated wake (right) where $V_{\mathrm{x}}$ is the local axial velocity and $V_{\mathrm{s}}$ is the ship's advance speed 


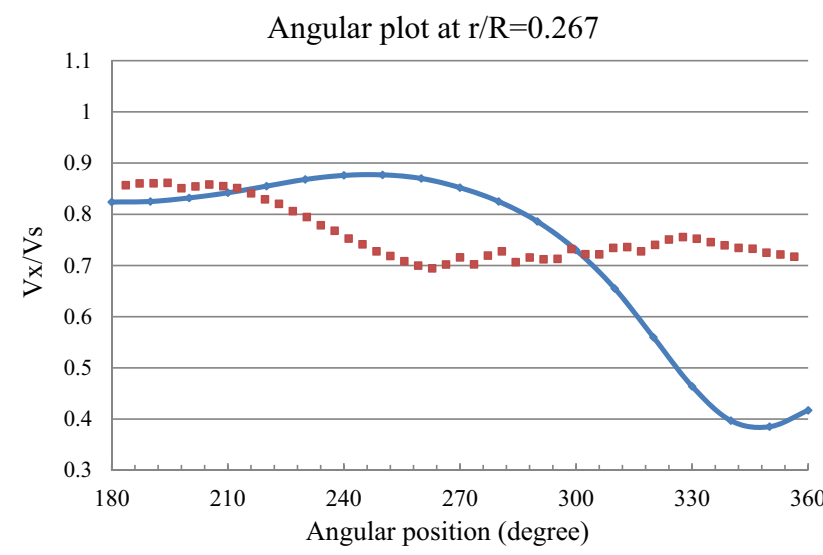

-ITU model test

- Final mesh with rudder

Fig. 27 Final wake comparison with rudder at $r / R=0.267$

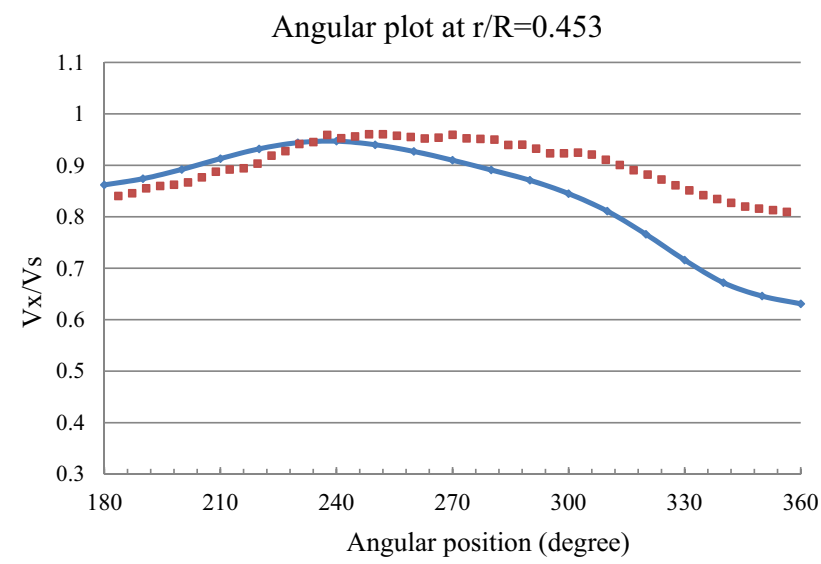

$\longrightarrow$ ITU model test

- Final mesh with rudder

Fig. 28 Final wake comparison with rudder at $r / R=0.453$

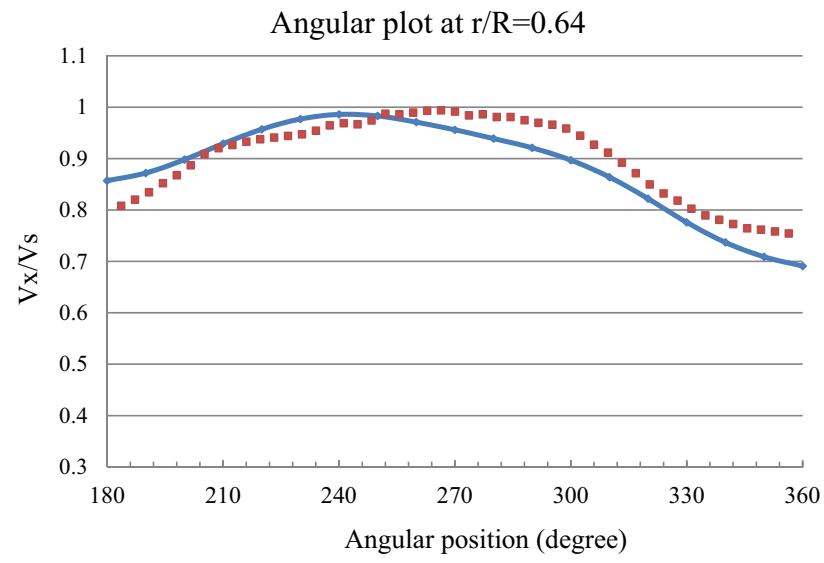

-ITU model test - Final mesh with rudder

Fig. 29 Final wake comparison with rudder at $r / R=0.64$

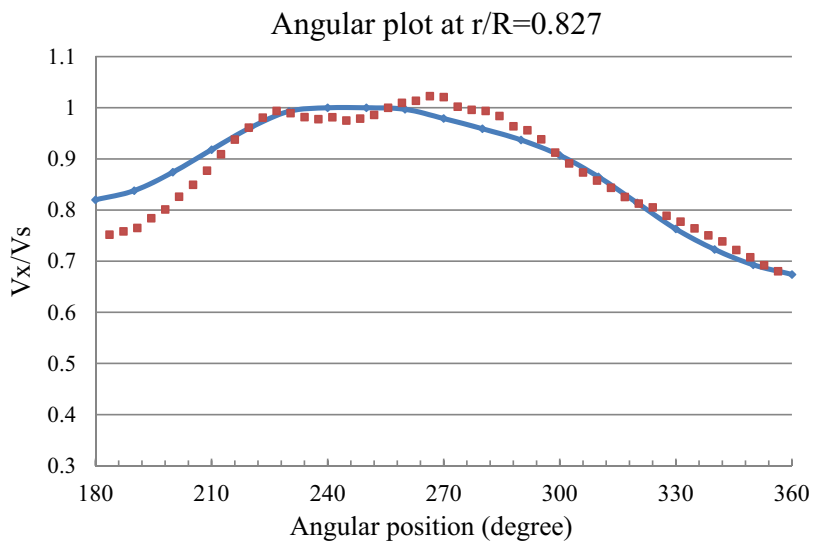

_ITU model test $\quad$ - Final mesh with rudder

Fig. 30 Final wake comparison with rudder at $r / R=0.827$

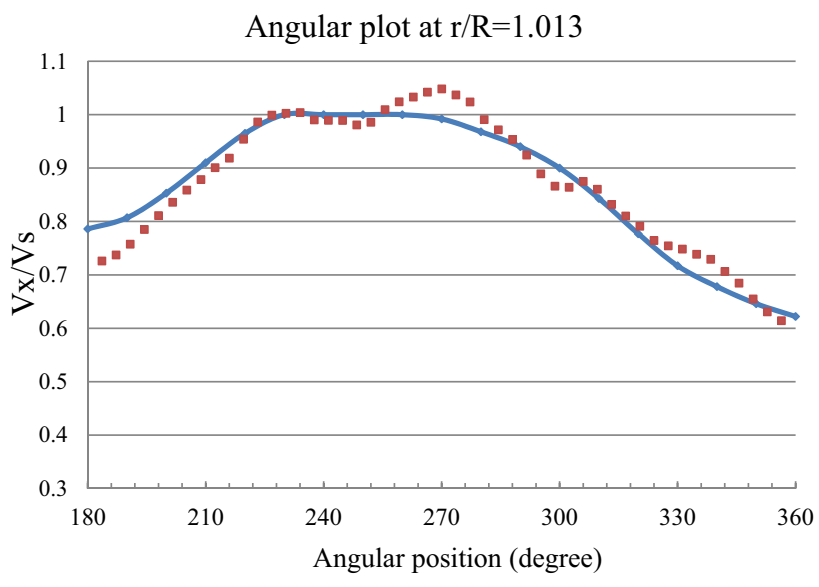

—ITU model test - Final mesh with rudder

Fig. 31 Final wake comparison with rudder at $r / R=1.013$

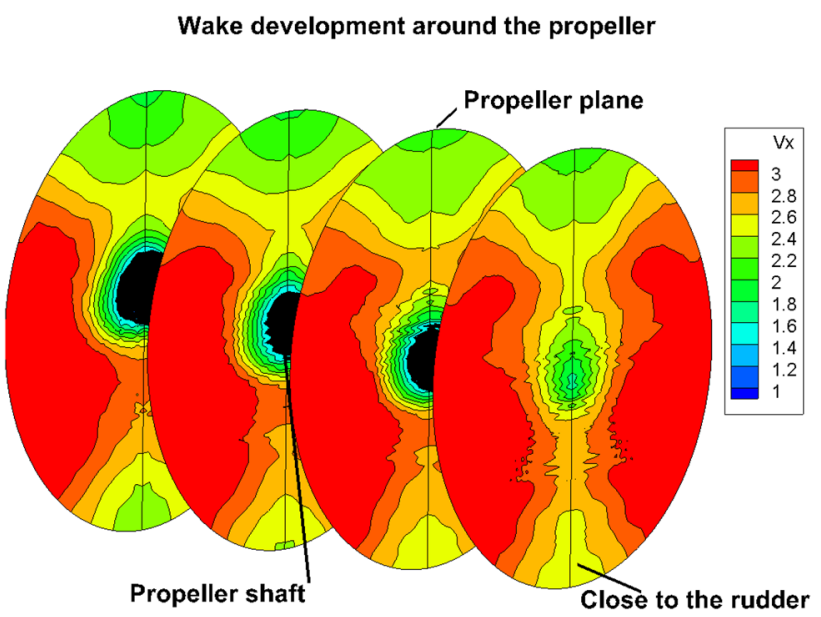

Fig. 32 Wake development of the dummy-hull model using SPIV flow measurements 


\section{Conclusions}

The wake distribution at the stern of a ship is critical for the propeller cavitation and noise measurements. This paper presents a relatively efficient wake simulation method for a shortened dummy hull model in a medium-sized cavitation tunnel using SPIV. The main conclusions are shown below.

1. A novel approach based on SPIV system for streamline tracing is presented in this paper. By the aid of this method, the consequent effect of the patch added to the wake screen and its influence at the propeller plane can be figured out so that the construction of wake screen can be more efficient. While normal wake simulation may take up to 10 mesh patch modifications, satisfactory resemblance of the target wake was achieved with only three attempts for this particular case.

2. The wake screen is constructed around 1.5 diameters before propeller plane. After adding three patches onto the wake screen, the wake distribution was matched to the target wake distribution. The agreements for radii $r / R>0.453$ are particularly encouraging to meet the expectations for the following propeller cavitation and noise test.

3. A series of wake field measurements were also conducted for upstream and downstream of the propeller to observe the flow development. According to these tests, the wake shadow area is gradually decreasing from the stern to the propeller plane and increasing after the propeller plane because of the existing rudder.

Acknowledgements This project was supported by the School of Marine Sciences and Technology as part of EU 7th Framework Programme (FP7) SONIC Project under Grant agreement no. 314394. The authors thank Dr. Rod Sampson, for his help during the experiments.

Open Access This article is distributed under the terms of the Creative Commons Attribution 4.0 International License (http://creativecommons.org/licenses/by/4.0/), which permits unrestricted use, distribution, and reproduction in any medium, provided you give appropriate credit to the original author(s) and the source, provide a link to the Creative Commons license, and indicate if changes were made.

\section{References}

1. Ross D (1976) Mechanics of underwater noise. Peninsula Publishing, California. doi:10.1016/B978-0-08-021182-4.50014-3

2. Reynolds III JE, Perrin WF, Reeves RR, Montgomery S, Ragen TJ (eds) (2005) Marine mammal research conservation beyond crisis. The Johns Hopkins University Press, Baltimore

3. IMO (2011) Noise from commercial shipping and its adverse impact on marine life-development of an international standard for measurement of underwater noise radiated from merchant ships, MEPC 62nd session Agenda item 19

4. IMO (2010) Noise from commercial shipping and its adverse impacts on marine life, Report of the Correspondence Group, MEPC 61st session Agenda item 19

5. Bertschneider H, Bosschers J, Choi GH, Ciappi E, Farabee T, Kawakita C, Tang D (2014) Specialist committee on hydrodynamic noise. In: Final report and recommendations to the 27 th ITTC. Copenhagen, Sweden, p 45

6. Atlar M, Aktas B, Sampson R, Seo K-C, Viola IM, Fitzsimmons P, Fetherstonhaugh C (2013) A multi-purpose marine science and technology research vessel for full-scale observations and measurements. In: International conference on advanced model measurement technologies for the Maritime industry (AMT'13), Gdansk, Poland

7. Aktas B, Atlar M, Turkmen S, Shi W, Sampson R, Korkut E, Fitzsimmons P (2016) Propeller cavitation noise investigations of a research vessel using medium size cavitation tunnel tests and full-scale trials. Ocean Eng 120:122-135. doi:10.1016/j. oceaneng.2015.12.040

8. Harvald SA (1981) Wake distributions and wake measurements. In: Transactions of the Royal Institution of Naval Architects, vol 123, pp 265-286

9. Holden K (1981) Effect of propeller design parameters on noise induced by cavitation. In: Nilsson AC, Tyvand NP (eds) Noise sources in ships I: propellers. Nordforsk, Miljovardsserien, Sweden

10. Sharma SD, Mani K, Arakeri VH (1990) Cavitation noise studies on marine propellers. J Sound Vib 138:255-283. doi:10.1016/0022-460X(90)90542-8

11. Odabasi A, Fitzsimmons P (1978) Alternative methods for wake quality assessment. Int Shipbuild Prog 25(282):8

12. Konno A, Wakabayashi K, Yamaguchi H, Maeda M, Ishii N, Soejima S, Kimura K, Yamguchi H, Maeda M, Ishii N, Soejima S, Kimura K (2002) On the mechanism of the bursting phenomena of propeller tip vortex cavitation. J Mar Sci Technol 6:181-192. doi:10.1007/s007730200006

13. ITTC (2011) ITTC — recommended procedures and guidelinesexperimental wake scaling methods. In: 7.5-02-03-02.5, p 7

14. ITTC (2011) Model—scale cavitation test. In: Recommended procedures guidelines 7.5-02-03-03.1, 26th ITTC specialist committee on scaling of wake fields, p 9

15. Atlar M, Takinaci AC, Korkut E, Aono T, Sasaki N (2001) Cavitation tunnel tests for propeller noise of a FRV and comparisons with full-scale measurements. In: International symposium cavitation, CAV2001 session B8.007, California Institute of Technology, Pasadena, CA, USA, p 13. http://caltechconf. library.caltech.edu/104/. Accessed 2 May 2014

16. ITTC (2011) The specialist committee on scaling of wake field. In: Final reports and recommendations to 26th ITTC, vol II, Rio de Janeiro, Brazil, 28 August-3 September. http://www.ittc.info/ media/5530/10.pdf. Accessed 8 May 2017

17. Sasajima H, Tanaka I (1966) On the estimation of wakes of ships. In: Proceedings of the 11th (ITTC) international towing tank conference. Society of Naval Architects and Marine Engineers, Tokyo, Japan, 11-20 October 1966

18. Hoekstra M (1975) Prediction of full scale wake characteristics based on model wake survey. Int Shipbuild Prog. https://trid.trb. org/view.aspx?id=40179. Accessed 26 Apr 2017

19. van Wijngaarden E (2011) Prediction of propeller-induced hullpressure fluctuations, 25 November, PhD, TU Delft. http://www. marin.nl/web/Publications/Publication-items/Prediction-of-PropellerInduced-HullPressure-Fluctuations.htm. Accessed 2 May 2014 
20. Lee SJ, Koh MS, Lee CM (2003) PIV velocity field measurements of flow around a KRISO 3600TEU container ship model. J Mar Sci Technol 8:76-87. doi:10.1007/s00773-003-0156-4

21. Lee JY, Paik BG, Lee SJ (2009) PIV measurements of hull wake behind a container ship model with varying loading condition. Ocean Eng 36:377-385. doi:10.1016/j.oceaneng.2009.01.006

22. Atlar M (2011) Recent upgrading of marine testing facilities at Newcastle University. In: 2nd International conference on advanced model measurement technology EU Maritime Industry, Newcastle upon Tyne, UK, 4-6 April, pp 1-32

23. Korkut E, Takinaci AC (2013) $18 \mathrm{M}$ research vessel wake measurements. Istanbul Technical University, Faculty of Naval Architecture and Ocean Engineering, İstanbul, p 6
24. Korkut E, Atlar M (2002) On the importance of the effect of turbulence in cavitation inception tests of marine propellers. Proc $\mathrm{R}$ Soc A Math Phys Eng Sci 458:29-48. doi:10.1098/rspa.2001.0852

25. Korkut E (1999) An investigation into the scale effects on cavitation inception and noise in marine propellers. School of Marine Science and Technology, Newcastle University, Philosophy of Doctorate Thesis

26. ANSI (2009) Quantities and procedures for description and measurement of underwater sound from ships-part 1: general requirements. Acoustical Society of America. doi:10.1016/0003-6870(91)90314-8 